\title{
MINIMUM THRUST OF A MORPHING UNMANNED SUBMERSIBLE AERIAL VEHICLE IN THE WATER-TO-AIR MOTION
}

\begin{abstract}
Summary
This study proposes a new water-to-air motion pattern that combines morphing with power switch. Under the conditions of this pattern, the vehicle needs a certain thrust to avoid falling back after jumping out of the water. The minimum thrust is among the most important design parameters of a vehicle. The water-exit and take-off dynamic models of the vehicle are constructed through the force and motion analysis before and after morphing. The control model of the vehicle is created by analysing the control problem in the take-off motion. The minimum thrust at different initial water-exit angles is computed using the optimum searching algorithm. The following law is then established: the greater the initial water-exit angle, the smaller the minimum thrust required in the air. Such a relationship becomes insignificant when the initial water-exit angle exceeds $40^{\circ}$.
\end{abstract}

Key words: $\quad$ Morphing Unmanned Submersible Aerial Vehicle (MUSAV), water-to-air motion, minimum thrust, optimization algorithm

\section{Introduction}

A vehicle that can make an easy transition from water to air was proposed as early as 1934 [1]. This vehicle which can submerge in the water and fly in the air has been extensively studied and debated by researchers. This vehicle concept has also been cited in several reports, such as the "LPL" project of the Soviet Union, the large submersible aircraft scheme of the United States, the conceptual design of a submersible aircraft by DARPA, and the "Aelius" AUV of France [1]. The Flimmer (Flying Swimmer) program of the Naval Research Laboratory has recently introduced a novel airborne delivery method for unmanned underwater vehicles [2].

Among the major technological difficulties in the water-exit motion are the significant differences in the physical properties of water and air. Present studies on launching carriers out of the water via submarines are scarce. The water-exit motion of a cylindrical body and the interaction between the free surface and cavity have been simulated [3], thereby capturing 
an increase in pressure as a result of the collapse of cavities. On the basis of the homogeneous multi-phase flow model, Cao [4] performed a numerical simulation of the collapse of a cavity filled with vapour and air, and found that the nose shape with a stronger cavitation capability can easily transfer the air in the launching tube into the low-pressure cavity. Xu [5] created a water-exit movement model for the dolphin leap of a torpedo. The simulation results confirm the validity of the proposed model and the solution for controlling the initial rotational angular velocity.

Some studies have investigated submarine-launched missiles and UAVs. Weiland [6] introduced the structure and the principle of a water-piercing missile launcher (WPML). Based on empirical evidence and numerical simulations, the WPML concept is established as a viable launcher concept for underwater bodies. Zink [7] investigated the splashdown of Cormorant UAVs into water using the methods of computational fluid dynamics and finite element analysis. A detailed design objective of submersible aircraft has been proposed in two reports $[8,9]$. The concept design aims at finding a solution to the problem of creating a vessel capable of multi-modal operations (airborne, surface, and submerged) and can make an easy transition between these modes. Various technologies, including the ones being developed, have been considered. For example, Bašić [10] proposed the Lagrangian method called incompressible smoothed particle hydrodynamics for simulating the dynamics of an incompressible viscous fluid flow. The obtained numerical results are in good agreement with the experimental and analytical findings in literature.

Submarine-launched missiles and UAVs are mainly studied by means of numerical and experimental methods. Previous studies have mainly focused on a particular condition, such as the vertical water-exit movement of flow field and load calculation. However, only few studies have investigated the entire trajectory design and its features. Furthermore, the present studies on submarine-launched UAVs have mostly investigated the water-exit motion using a qualitative method other than a quantitative analysis. Submersible aerial vehicles also need further study.

This study theoretically investigates the water-to-air motion of a morphing unmanned submersible aerial vehicle (MUSAV) that can submerge in the water and fly in the air. The water-to-air motion is divided into the water-exit stage and the take-off stage. First, the MUSAV will rely on inertia to move out of the water. Second, the vehicle will open its wings through a morphing movement and will simultaneously adjust the operating mode of the engine. Third, the vehicle will accelerate for the take-off under the control of angular moment. This study also proposes hydrodynamic and aerodynamic models of the vehicle that obliquely exits the water surface before the take-off. The control strategy of accelerating in the take-off stage has also been designed. The law of minimum thrust in different initial conditions has been obtained using the optimum searching algorithm.

\section{MUSAV and the water-to-air motion pattern}

\subsection{Shapes of the vehicle}

(1) Shape in water

A vehicle with an ogive nose and circular truncated cone tail is designed. Fig. 1 shows the contour of the vehicle in water. The length and density of the vehicle are assumed to be $L=5.33 \mathrm{~m}, \rho_{v}=1.2 \times 10^{3} \mathrm{~kg} / \mathrm{m}^{3}$, respectively and the mass is assumed to be uniformly distributed. 


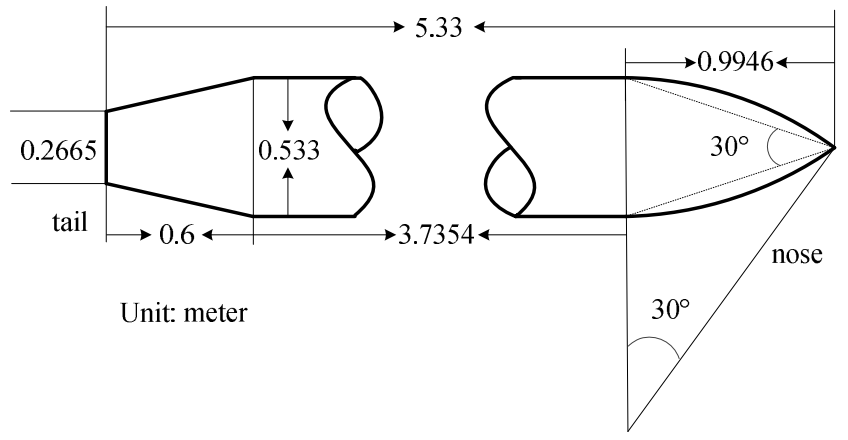

Fig. 1 Contour of the vehicle in water

(2) Shape in air

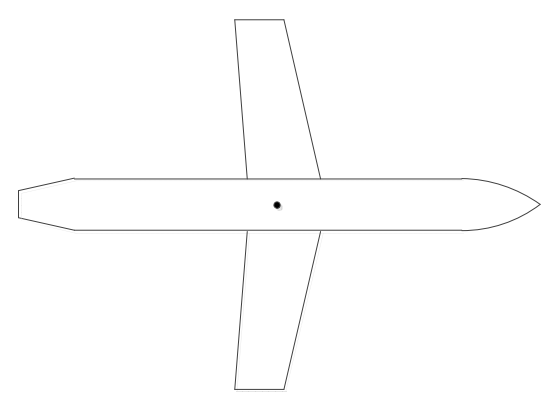

Fig. 2 Contour of the vehicle in air

Unlike its shape in water, the shape of the vehicle in air includes an additional pair of wings, as shown in Fig. 2. The mono-wing has an area of $1 \mathrm{~m}^{2}$. The air foil adopts a NASA GA(W)-1 high-lift air foil and NASA standard roughness. The drag acts directly on the centre of gravity of the vehicle.

The assumptions of this study are as follows: 1. morphing does not change the mass and centre of gravity of the vehicle; 2. the morphing gear and thrust can be realized in engineering; and 3. morphing is completed instantaneously, $0.5 \mathrm{~s}$ after the vehicle exits the water surface.

\subsection{Summary of water-to-air motion}

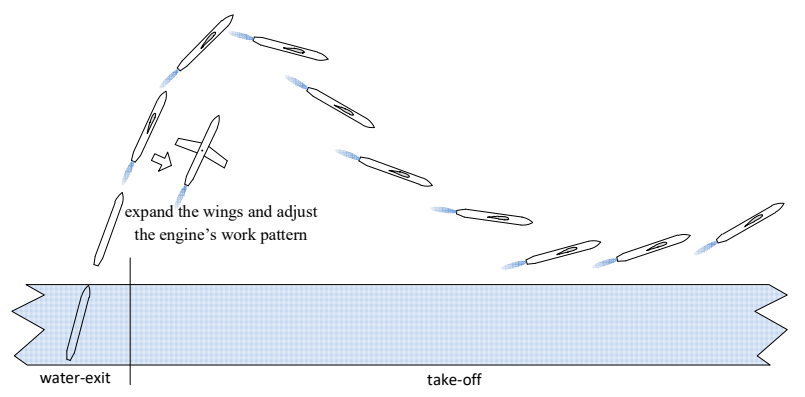

Fig. 3 The water-to-air motion

The entire water-to-air motion of a MUSAV can be divided into the water-exit stage and the take-off stage, as shown in Fig. 3. First, the vehicle jumps out of the water by means of inertia with the water shape. Second, the wings are opened and the engine starts to work. Third, the vehicle is controlled to avoid stalling as a result of the great attack angle, and then the vehicle descends continuously because of low speed. Finally, the speed increases by enhancing the thrust and using the control strategy, thereby providing the vehicle with sufficient ascensional force to climb timely before dropping into the water. 


\section{Motion model}

\subsection{Water-exit stage}

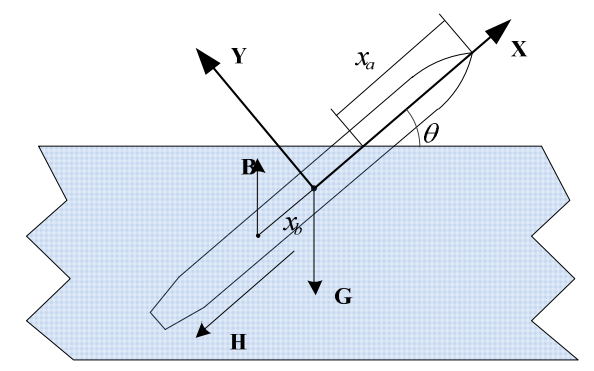

Fig. 4 Force analysis in the water-exit stage

Given the low velocity of the vehicle in the water-exit stage, the effect of air can be ignored. The vehicle is under the effect of only gravity $\mathbf{G}$, buoyancy $\mathbf{B}$, and fluid force $\mathbf{H}$, as shown in Fig. 4. In this figure, $\theta$ denotes the inclination angle, while $x_{b}$ denotes the distance between the mass centre and the buoyancy centre.

According to the vector and the moment of ideal fluid acting on the vehicle [11], the definition of added mass [12] and the ideal fluid force equation can be written as follows. The change rate of the added mass is considered in this study.

$$
\left\{\begin{aligned}
H_{i x}= & -\lambda_{11} \dot{v}_{x}-\dot{\lambda}_{11} v_{x}+\omega_{z}\left(\lambda_{22} v_{y}+\lambda_{26} \omega_{z}\right) \\
H_{i y} & =-\lambda_{22} \dot{v}_{y}-\dot{\lambda}_{22} v_{y}-\lambda_{26} \dot{\omega}_{z}-\dot{\lambda}_{26} w_{z}-\omega_{z} \lambda_{11} v_{x} \\
M_{i z} & =-\lambda_{62} \dot{v}_{y}-\dot{\lambda}_{62} v_{y}-\lambda_{66} \dot{\omega}_{z}-\dot{\lambda}_{66} w_{z}+v_{y} \lambda_{11} v_{x} \\
& \quad-v_{x}\left(\lambda_{22} v_{y}+\lambda_{26} \omega_{z}\right)
\end{aligned}\right.
$$

where $H_{i x}$ and $H_{i y}$ are the ideal fluid forces in the $\mathrm{X}$ and $\mathrm{Y}$ directions, respectively; $M_{i z}$ is the moment; $v_{x}$ and $v_{y}$ are the velocities in the $\mathrm{X}$ and $\mathrm{Y}$ directions, respectively; $\omega_{z}$ is the angular velocity; and $\lambda$ is the added mass.

The profile analysis method is adopted as follows to calculate the added mass [13]:

$$
\left\{\begin{array}{l}
\lambda_{22}=\pi \rho_{v} \int_{0}^{L-x_{a}} R^{2}(x) \mathrm{d} x \\
\lambda_{26}=\pi \rho_{v} \int_{0}^{L-x_{a}} R^{2}(x) x \mathrm{~d} x \\
\lambda_{66}=\pi \rho_{v} \int_{0}^{L-x_{a}} R^{2}(x) x^{2} \mathrm{~d} x
\end{array}\right.
$$

where $\rho_{v}$ is the density of the vehicle, $L$ is the length of the vehicle, $x$ is the distance between one point on the symmetry axis of the vehicle and the tail, $R(x)$ is the radius at this point, and $x_{a}$ is the length of the front part of the vehicle outside of the water surface as shown in Fig. 4. The following equation shows that $\mathrm{d} x_{a}=v_{x} \mathrm{~d} t$. Given that the slender body has a very low added mass $\lambda_{11}$ [13], $\lambda_{11}$ and $\dot{\lambda}_{11}$ are both equal to 0 . 


$$
\left\{\begin{array}{l}
\mathrm{d} \lambda_{22}=-\pi \rho_{v} R^{2} \mathrm{~d} x_{a} \\
\mathrm{~d} \lambda_{26}=-\pi \rho_{v}\left(L-x_{a}\right) R^{2} \mathrm{~d} x_{a} \\
\mathrm{~d} \lambda_{66}=-\pi \rho_{v}\left(L-x_{a}\right)^{2} R^{2} \mathrm{~d} x_{a}
\end{array}\right.
$$

The drag by [5] is expressed as:

$$
F_{\mu}=C_{x} \cdot \frac{1}{2} \rho_{w} v^{2} S
$$

The force caused by viscous fluid and the attack angle is shown below:

$$
\left\{\begin{array}{l}
H_{\mu x}=-F_{\mu} \cdot \cos \alpha \\
H_{\mu y}=F_{\mu} \cdot \sin \alpha \\
M_{\mu z}=H_{\mu y} \cdot \frac{1}{2} x_{a}
\end{array}\right.
$$

where $H_{\mu x}$ is the drag caused by the viscous fluid, $H_{\mu y}$ and $M_{\mu z}$ are the lift and pitch moments caused by the attack angle of the vehicle, $\rho_{w}$ is the density of water, $v$ is the velocity of the vehicle, $S$ is the wetted area of the vehicle, and $\alpha$ is the attack angle.

Based on the above force analysis, the water-exit stage dynamic model is established in the body coordinate system of the vehicle:

$$
\left\{\begin{array}{l}
H_{i x}+H_{\mu x}+(B-G) \sin \theta=m\left(\mathrm{~d} v_{x} / \mathrm{d} t-v_{y} \omega_{z}\right) \\
H_{i y}+H_{\mu y}+(B-G) \cos \theta=m\left(\mathrm{~d} v_{y} / \mathrm{d} t+v_{x} \omega_{z}\right) \\
M_{i z}+M_{\mu z}-B x_{b} \cos \theta=J \cdot \mathrm{d} \omega_{z} / \mathrm{d} t
\end{array}\right.
$$

where $\theta$ is the inclination angle, $x_{b}$ is the distance between the mass centre and the buoyancy centre, and $J$ is the moment of inertia of the vehicle.

Therefore, when the initial state $X_{0}=\left(x_{0}, y_{0}, v_{x 0}, v_{y 0}, \omega_{z 0}\right)$ of the vehicle is given, the end state $X_{\text {out } 0}$ after the vehicle jumps out of the water can be obtained according to Eqs. (1)-(6). We use $X_{\text {out } 0}=U_{1}\left(X_{0}\right)$ to express this computation process.

\subsection{Take-off stage}

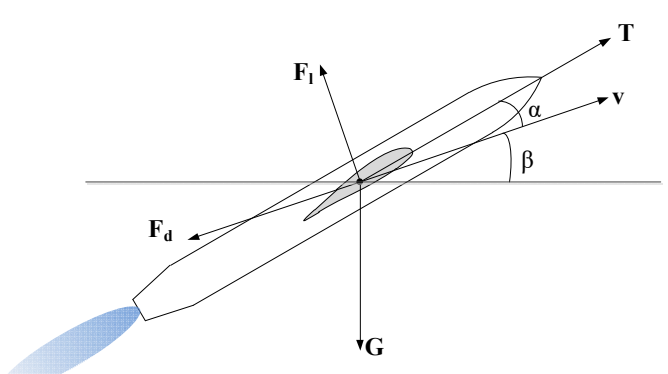

Fig. 5 Force analysis in the take-off stage

In the take-off stage, the vehicle is under the effect of gravity $\mathbf{G}, \operatorname{lift} \mathbf{F}_{l}$, drag of air $\mathbf{F}_{d}$, thrust $\mathbf{T}$, and control moment $M_{z}$, as shown in Fig. 5.

The lift, drag, and pitching moments of the air can be defined by dividing the air into wing and fuselage. 
1. Wing

The wing lift $F_{l w}$, the wing drag $F_{d w}$, and the wing pitching moment $M_{w}$ of air can be defined using the following lift and drag equations:

$$
\left\{\begin{array}{l}
F_{l w}=\frac{1}{2} C_{l w}(\alpha) \rho_{a} v^{2} S_{w} \\
F_{d w}=\frac{1}{2} C_{d w}(\alpha) \rho_{a} v^{2} S_{w} \\
M_{w}=\frac{1}{2} C_{w}(\alpha) \rho_{a} v^{2} S_{w}
\end{array}\right.
$$

where $C_{l w}(\alpha)$ is the wing lift coefficient, $C_{d w}(\alpha)$ is the wing drag coefficient, and $C_{w}(\alpha)$ is the wing pitching moment coefficient, $\rho_{a}$ is the density of air, and $S_{w}$ is the wing area.

The aero foil in this study adopts the NASA GA(W)-1 high-lift aero foil; Fig. 6 shows the distributions of the lift, drag, and pitching moment coefficients for the attack angle $\alpha \in\left(0^{\circ}, 20.5^{\circ}\right)[14]$.

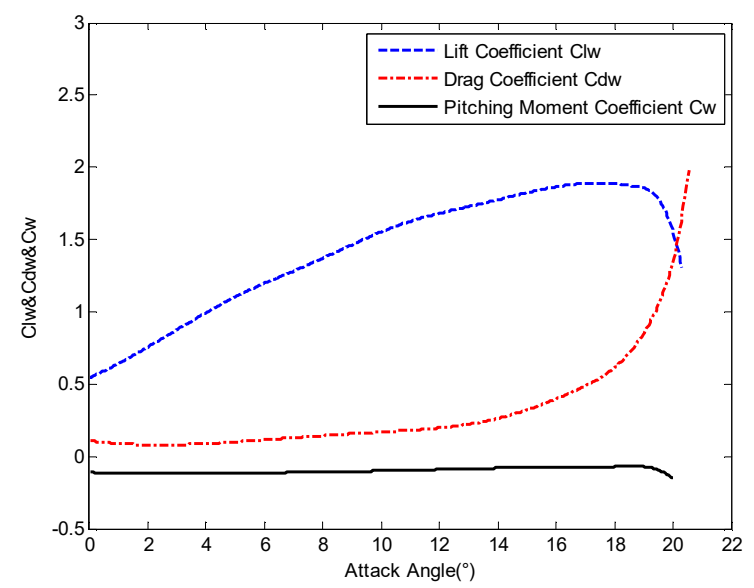

Fig. 6 Regularity of the wing lift, drag and pitching moment coefficients

\section{Fuselage}

The fuselage lift coefficient, fuselage drag coefficient, and fuselage pitching moment coefficient can be worked out by Fluent simulation.

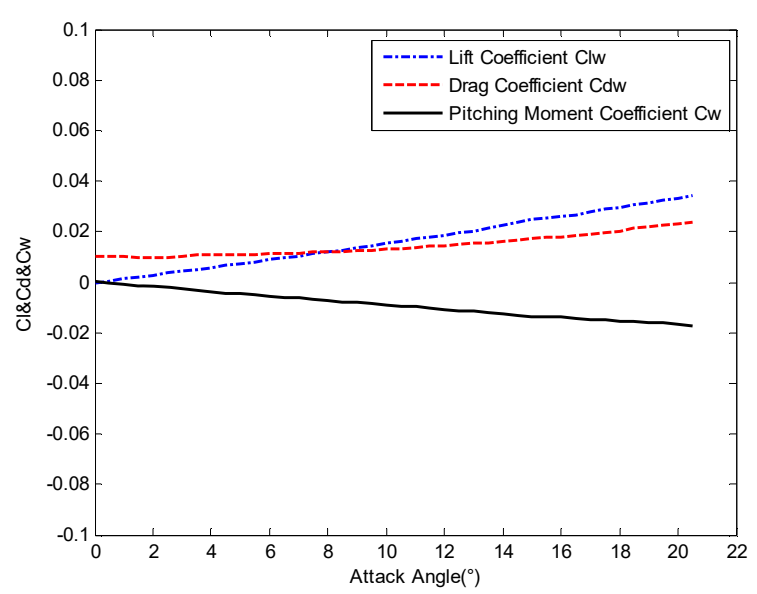

Fig. 7 Regularity of the fuselage lift, drag and pitching moment coefficients 
The fuselage lift, fuselage drag, and fuselage pitching moment coefficients can be obtained via Fluent simulation. The fuselage lift $F_{l f}$, fuselage drag $F_{d f}$, and fuselage pitching moment $M_{f}$ can then be obtained using the lift and drag equations:

$$
\left\{\begin{array}{l}
F_{l f}=\frac{1}{2} C_{l f}(\alpha) \rho_{a} v^{2} S_{f} \\
F_{d f}=\frac{1}{2} C_{d f}(\alpha) \rho_{a} v^{2} S_{f} \\
M_{f}=\frac{1}{2} C_{f}(\alpha) \rho_{a} v^{2} S_{f}
\end{array}\right.
$$

where $C_{l f}(\alpha)$ is the fuselage lift coefficient, $C_{d f}(\alpha)$ is the fuselage drag coefficient, $C_{f}(\alpha)$ is the fuselage pitching moment coefficient, $\rho_{a}$ is the density of air, and $S_{f}$ is the fuselage area.

The take-off stage dynamic model is created in the absolute coordinate system. The state of the vehicle can be defined as $X=\left(x, y, v, \beta, \alpha, \omega_{z}\right)$, where $x$ and $y$ are the absolute coordinates, $v$ is the velocity, $\beta$ is the inclination angle, $\alpha$ is the attack angle, and $\omega_{z}$ is the rotation angular velocity. Equation (9) is the state transition equation of $X$.

$$
\left\{\begin{array}{l}
\frac{\mathrm{d} x}{\mathrm{~d} t}=v \cdot \cos \beta \\
\frac{\mathrm{d} y}{\mathrm{~d} t}=v \cdot \sin \beta \\
\frac{\mathrm{d} v}{\mathrm{~d} t}=\frac{T \cos \alpha-F_{d w}-F_{d f}-m g \sin \beta}{m} \\
\frac{\mathrm{d} \beta}{\mathrm{d} t}=\frac{T \sin \alpha+F_{l w}+F_{l f}-m g \cos \beta}{m v} \\
\frac{\mathrm{d} \alpha}{\mathrm{d} t}=\omega_{z}-\frac{\mathrm{d} \beta}{\mathrm{d} t} \\
\frac{\mathrm{d} \omega_{z}}{\mathrm{~d} t}=\frac{M_{z}+M_{f}+M_{w}}{J_{z}}
\end{array}\right.
$$

where $J_{z}$ is the moment of inertia and $M_{z}$ is the input pitch moment that is supposed to be produced by a direct force. Installing an attitude control mechanism, one can control the attitude of the vehicle. This mechanism comprises several small engines, as shown in Fig. 8 . When needed, one or more engines in a certain direction can be started to provide the corresponding control moment. This study assumes that the attitude control mechanism produces a certain range of input moment. 


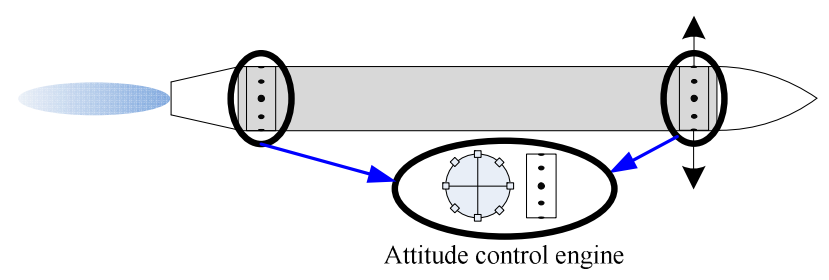

Fig. 8 Attitude control engine on the vehicle

If the initial state is given, then the model equation can be solved when the control thrust $\mathbf{T}$ and the moment $M_{z}$ are inputted into the model. The take-off stage computation process is expressed as $X_{t}=U_{2}\left(X_{\text {out } 0}, \mathbf{T}\right)$.

\section{Morphing and flight control}

When the vehicle jumps out of the water, the corresponding control is needed to achieve stable flying in the air. The attack angle must be selected according to the distribution of the lift and drag coefficients for the NASA GA(W)-1 profile. The control aims at maintaining the attack angle at $19^{\circ}$.

The maximum moment of control is assumed to be $500 \mathrm{~N} \cdot \mathrm{m}$.

For the state transition expressed in Eq. (9), the control input is the moment $M_{z}$, the control output is the attack angle $\alpha$, and the ideal control output is the ideal attack angle $\alpha_{0}=19^{\circ}$.

$$
\left\{\begin{array}{l}
u=M_{z} \\
C_{\text {out }}=\alpha \\
C_{\text {ideal }}=\alpha_{0}
\end{array}\right.
$$

For the relationship between the control output $C_{\text {out }}$ and the control input $u$,

$$
C_{\text {out }}^{\prime}=\alpha^{\prime}=\omega_{z}-\beta^{\prime}=\omega_{z}-\frac{T \sin \alpha+F_{l w}+F_{l f}-m g \cos \beta}{m v}
$$

we get

$$
\begin{aligned}
C_{\text {out }}^{\prime \prime} & =\omega_{z}^{\prime}-\left(\frac{T \sin \alpha+F_{l w}+F_{l f}-m g \cos \beta}{m v}\right)^{\prime} \\
& =\frac{M_{z}+M_{f}+M_{w}}{J_{z}}-\frac{T \alpha^{\prime} \cos \alpha+m g \beta^{\prime} \sin \beta}{m v}+\frac{\left(T \sin \alpha+F_{l w}+F_{l f}-m g \cos \beta\right) v^{\prime}}{m v^{2}}
\end{aligned}
$$

$$
\text { Assuming } f(X)=\frac{T \alpha^{\prime} \cos \alpha+m g \beta^{\prime} \sin \beta}{m v}+\frac{\left(T \sin \alpha+F_{l w}+F_{l f}-m g \cos \beta\right) v^{\prime}}{m v^{2}}, \text { Eq. }
$$

can be rewritten as:

$$
C_{\text {out }}^{\prime \prime}=\frac{M_{z}+M_{f}+M_{w}}{J_{z}}-f(X)=\left(u+M_{f}+M_{w}\right) / J_{z}-f(X)
$$


Then, the control law can be set as:

$$
u=(\eta+f(X)) J_{z}-M_{f}-M_{w}
$$

where $\eta$ is the auxiliary variable of the control law.

For $C_{\text {out }}^{\prime \prime}=\eta$, the error is $e=C_{\text {ideal }}-C_{\text {out }}$. For $\eta$ designed in the form of feedback linearization, we have

$$
\eta=C_{i d e a l}^{\prime \prime}+k_{1} e+k_{2} e^{\prime}
$$

Where, $k_{1}$ and $k_{2}$ are both positive real numbers. Therefore, $e^{\prime \prime}+k_{2} e^{\prime}+k_{1} e=0$. Because $k_{1}$ and $k_{2}$ are positive real numbers, then $t \rightarrow \infty, e \rightarrow 0$.

\section{Optimization algorithm for obtaining the minimum thrust}

To help a vehicle with different water-exit angles jump out of the water by means of a minimum thrust, the variation law of the necessary thrust for the vehicle to successfully take off must be investigated. Using this law, the minimum thrust of the vehicle can be obtained by controlling its different water-exit angles.

Analytic answers cannot be determined because of the complexity of the water-exit and take-off motion models, which are obtained from the multivariate differential equations of two intricate structures. An optimal solver model is therefore established to determine the minimum thrust for certain angles. This method can be converted to the constraint extreme value problem as follows:

$$
\begin{array}{ll}
\min \left(\min _{t}\left(d_{t}\right)\right) & =U\left(\theta_{\text {begin }}, T_{\text {th }}\right) \\
& =U_{2}\left(U_{1}\left(X_{0}\left(\theta_{\text {begin }}\right), T_{\text {th }}\right)\right. \\
\text { s.t. } & 0<\theta_{\text {begin }}<90^{\circ} ; \\
& T_{t h}>0 ; \\
& \min _{t}\left(d_{t}\right)>0 .
\end{array}
$$

where $\theta_{\text {begin }}$ is the initial water-exit angle, $T_{t h}$ is the thrust acting on the vehicle, $d_{t}$ is the altitude of the mass centre after the vehicle changes its shape in the take-off stage, $\min _{t}\left(d_{t}\right)$ denotes the minimum value of $d_{t}$ in the take-off stage, and $U()$ denotes the previously established water-exit and take-off motion models of the vehicle. The constraint condition $\min _{t}\left(d_{t}\right)>0$ suggests that the vehicle must stay above the water surface in the entire take-off motion, that is, the vehicle must be prevented from falling back into the water. With given $\theta_{\text {begin }}$ and $T_{t h}$, the motion model $U()$ can be used to determine $\min _{t}\left(d_{t}\right)$.

Based on the physical characteristics of the water-to-air motion of the vehicle, the vehicle must have a thrust value $T_{t h \_ \text {min }}$ upon exiting the water, at which the vehicle can take off very close to the water surface before falling back into water. When $T_{t h}>T_{t h}$ min , the vehicle can take off normally. When $T_{t h}<T_{t h \_ \text {min }}$, the thrust acting on the vehicle is too low, thereby pushing the vehicle back into the water. The above optimal problem can be categorized as a unimodal function; such a problem can be solved by using the golden section search method [15]. 


\section{Computer simulations}

Simulation 1, simulation of water-exit movement. In the initial condition, the water-exit angle ranges between $20^{\circ}$ and $80^{\circ}$, the water-exit velocity is $20 \mathrm{~m} / \mathrm{s}$, and the water-exit angular speed is 0 . The water-exit process is solved using the variable-step Runge-Kutta algorithm. Fig. 9 shows the simulation under this condition. The simulation is based on the previously established water-exit motion model. The stop time in Fig. 9(a) refers to the moment when the tail of the vehicle leaves the water surface. The simulation in Fig. 9(b) is completed in $0.5 \mathrm{~s}$ after the simulation in Fig. 9(a). In Fig. 9, the heavy line denotes the location trajectory of the centre of mass of the projectile, the dotted line indicates the water surface, and the fine line denotes the profile of the projectile at different times. In Fig. 9(a), the entire water-exiting process of the vehicle is shown in 10 equal time points. Various simulation time intervals are adopted under different simulation conditions. The entire waterexiting time is considered, and each interval lasts for approximately $0.035 \mathrm{~s}$. In Fig. 9(b), the entire water-exiting process and the post-water-exit period of $0.5 \mathrm{~s}$ under different simulation conditions are shown in five equal time points. Each interval lasts for approximately $0.2 \mathrm{~s}$.

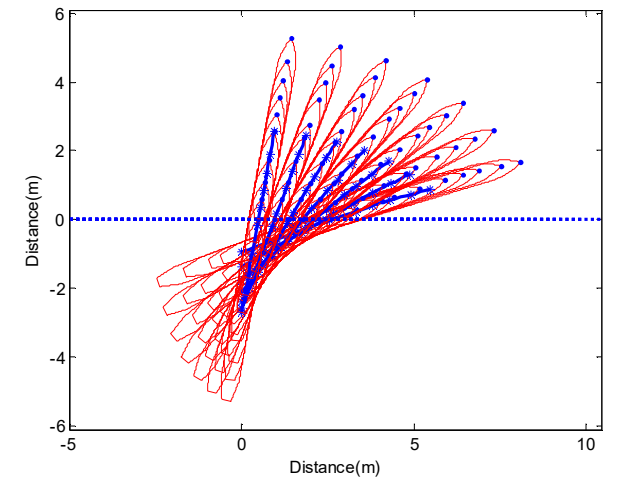

(a)

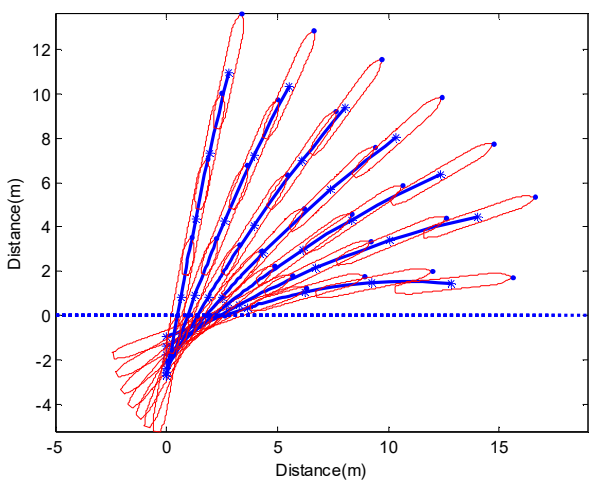

(b)

Fig. 9 Simulation of water-exit stage

Simulation 2, simulation of water-to-air movement. In the initial condition, the waterexit angle is $50^{\circ}$, the water-exit velocity is $20 \mathrm{~m} / \mathrm{s}$, and the water-exit angular speed is 0 . The water-to-air process simulation is solved using the constant-step Runge-Kutta algorithm. The time step is set to $0.01 \mathrm{~s}$. Under this condition, the golden section computes the minimum thrust as $13425.32 \mathrm{~N}$ after 10 iterations. Figs. 10-14 show the simulations under the minimum thrust. Fig. 10(b) shows further details about the water-exit motion presented in Fig. 10(a).

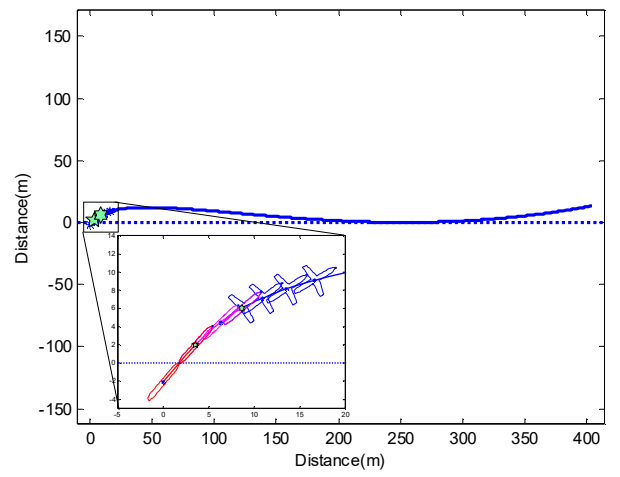

(a)

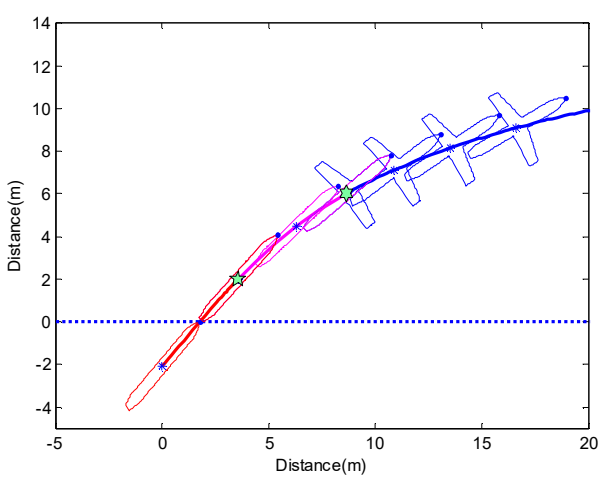

(b)

Fig. 10 Simulation of water-exit, morphing and take-off 


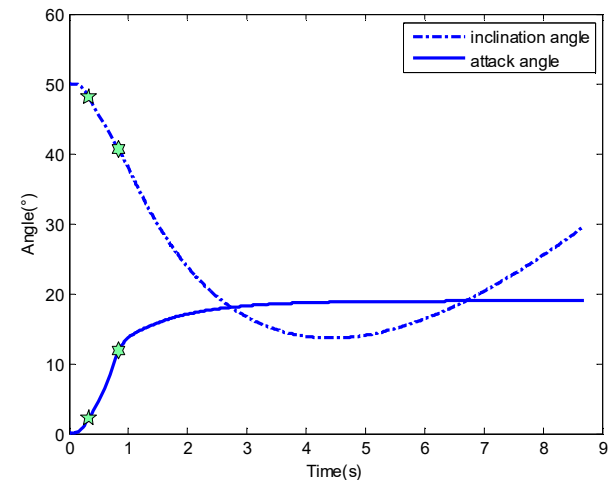

Fig. 11 Variations in the pitch angle and the attack angle

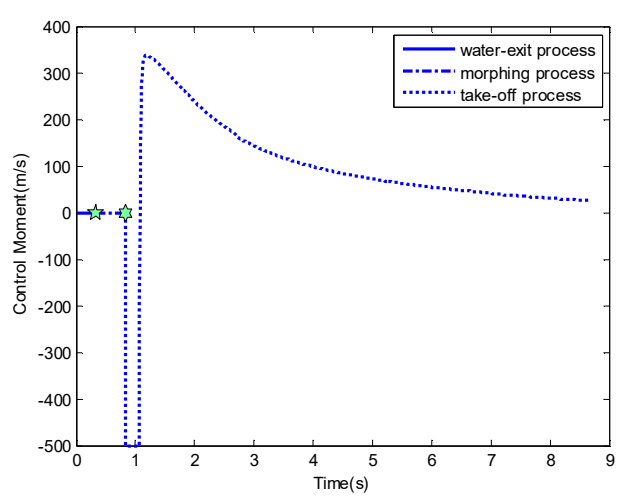

Fig. 13 Variations in the control input momentum

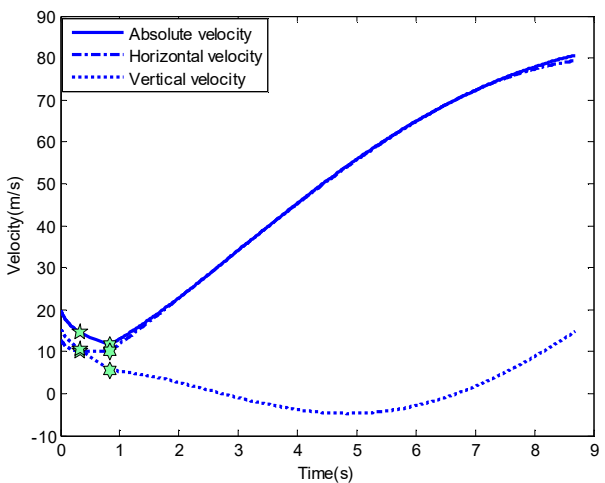

Fig. 12 Variation in velocity

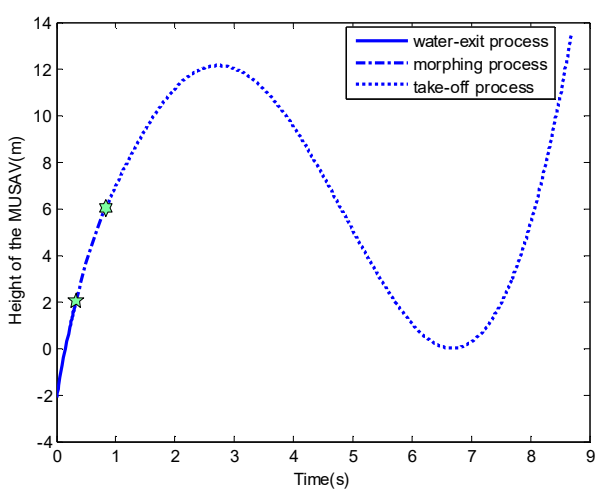

Fig. 14 Variation in altitude

Fig. 10 presents the outline of the vehicle at important moments. The time interval between the two outlines of the vehicle after variation is $0.2 \mathrm{~s}$. The pentagram in Figs. 10-14 denotes the moment at which the tail of the vehicle leaves the water surface, while the hexagon represents the moment at which the vehicle starts to change its shape. Figs. 10 and 14 show that the vehicle flies $250 \mathrm{~m}$ in about $6.8 \mathrm{~s}$ and then falls to the lowest point while staying close to the water surface. With the help of thrust and control, the vehicle climbs up gradually and then starts to take off. In Fig. 11, the attack angle slowly reaches $19^{\circ}$, thereby validating the availability of the control method. Fig. 13 shows the variations in the control input momentum.

Simulation 3. With a water-exit angle of $50^{\circ}$, the optimal search algorithm is used to derive the minimum thrust, which is shown in simulation 2 . The aim is to analyse the relationship between the necessary minimum thrust for the vehicle to exit water and the water-exit angle. Hence, the optimal search algorithm is used to derive the minimum thrust at different water-exit angles and a simulation is performed under the condition with the least thrust.

Table 1 is the result of the minimum thrust at different water-exit angles calculated by the optimal search algorithm.

Table 1 Minimum thrust at different water-exit angles

\begin{tabular}{ccccccc}
\hline water-exit angle $/{ }^{\circ}$ & 25 & 30 & 35 & 40 & 45 & 50 \\
minimum thrust $/ \mathbf{N}$ & 88357.55 & 32181.96 & 19695.85 & 16221.96 & 14565.91 & 13425.32 \\
\hline $\begin{array}{c}\text { water-exit angle } /{ }^{\circ} \\
\text { minimum thrust } / \mathbf{N}\end{array}$ & 55 & 60 & 65 & 70 & 75 & 80 \\
\hline
\end{tabular}


F. Jinfu, L. Yongli

X. Baowei, L. An, Q. Duo
Minimum Thrust of a Morphing Unmanned Submersible Aerial Vehicle in the Water-to-Air Motion

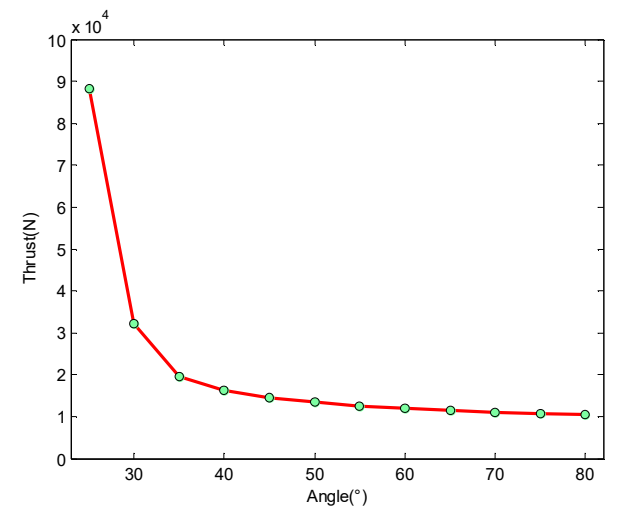

Fig. 15 Minimum thrust at different water-exit angles

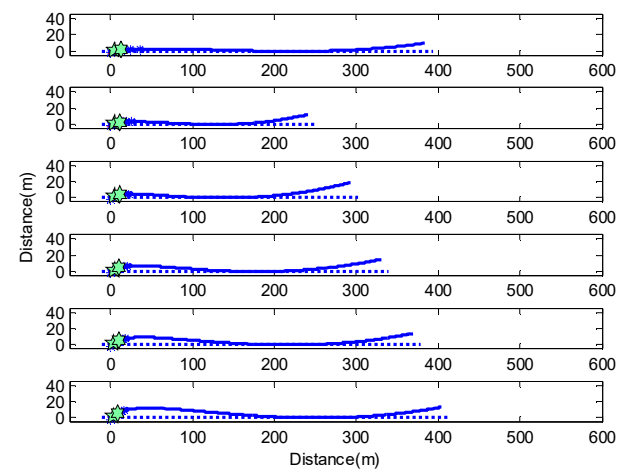

(a) $\theta_{\text {begin }}=25^{\circ}, 30^{\circ}, 35^{\circ}, 40^{\circ}, 45^{\circ}, 50^{\circ}$

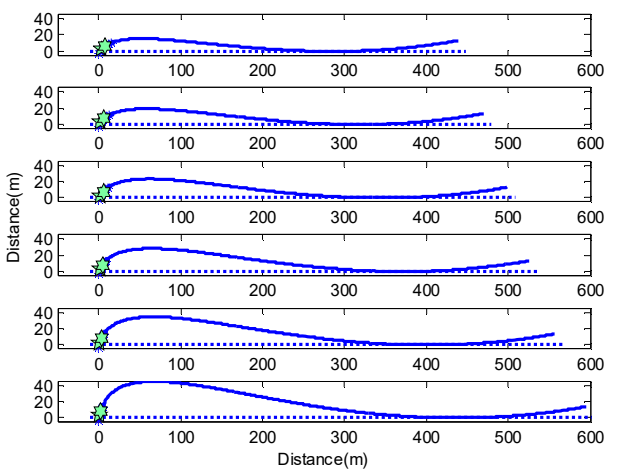

(b) $\theta_{\text {begin }}=55^{\circ}, 60^{\circ}, 65^{\circ}, 70^{\circ}, 75^{\circ}, 80^{\circ}$

Fig. 16 Simulations of minimum thrust

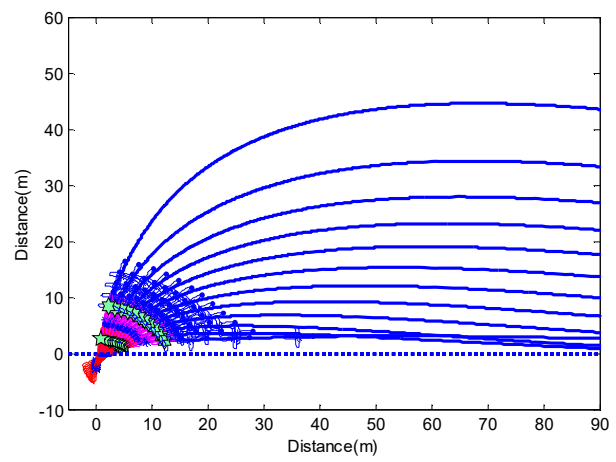

(a)

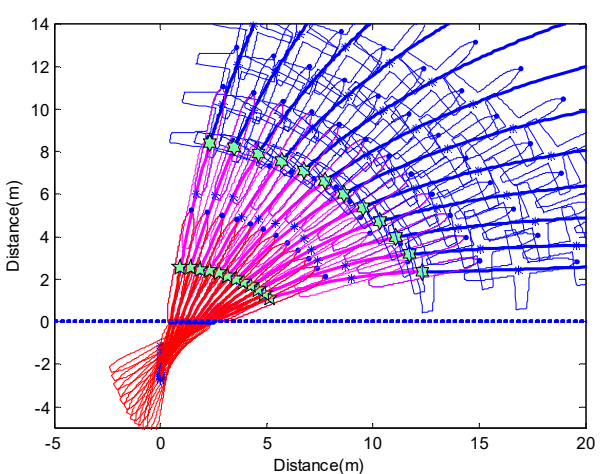

(b)

Fig. 17 Trajectory simulation of different angles

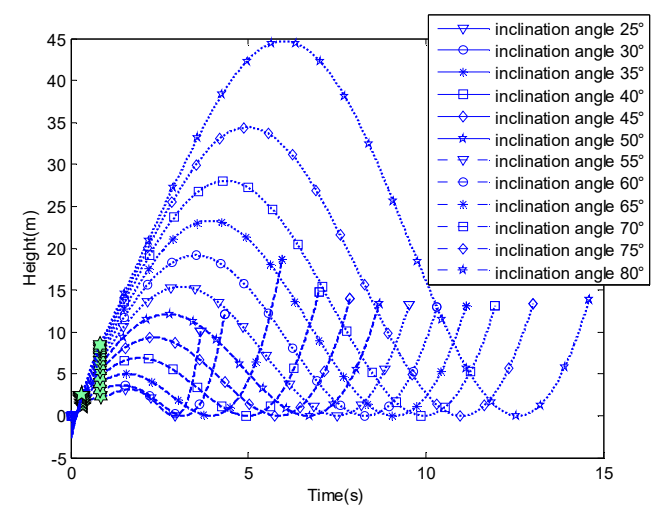

Fig. 18 Variations in the vehicle altitude at different initial water-exit angles 
Fig. 17(b) shows further details about the water-exit motion presented in Fig. 17(a). Fig. 17 presents the outline of the vehicle at important moments with different angles. The interval of the vehicle outline is not fixed in the simulations.

Fig. 15 shows that a greater water-exit angle indicates a lesser minimum thrust in the air. The simulation outcome shows that when the water-exit angle is greater than $40^{\circ}$, the variation curve of the minimum thrust tends to be horizontal. In other words, the water-exit angle of the vehicle must be greater than $40^{\circ}$ to reduce the minimum thrust in the air and must have as big measure as possible. However, the controllability of the vehicle must also be considered. A big measure of the water-exit angle increases the difficulty of the control. In the take-off stage, such difficulty may cause the attack angle of the vehicle to exceed the normal drag attack angle range, thereby stalling the vehicle. Figs. 15-18 show that the water-exit angle of the vehicle must range from $40^{\circ}$ to $80^{\circ}$. When the water-exit angle is less than $40^{\circ}$, the minimum thrust for the vehicle to take off will increase, thereby demanding great engine power. When the water-exit angle is greater than $80^{\circ}$, the attitude of the vehicle cannot be easily controlled from the post-water-exit period to horizontal flight, thereby increasing the attack angle beyond the critical value.

\section{Conclusion}

This study presents a novel method for realizing the water-to-air motion of a MUSAV. After leaving the water surface, the vehicle requires a certain thrust to take off instead of falling back into the water. This study also presents a dynamic model for the water-exit and take-off stages of the vehicle as well as its underwater and aerial shapes. The control mechanism of the vehicle for the take-off has also been analysed and devised. Using the optimal research algorithm, the minimum thrust is studied at a certain water-exit angle. On this basis, the relationship between the minimum thrust and the water-exit angle is analysed. The necessary thrust is appropriate and moderate when the water-exit angle ranges between $40^{\circ}$ and $80^{\circ}$. The simulation results prove the feasibility of the proposed method, the availability of flight control, and the applicability of the optimization algorithm.

Furthermore, the authors would like to thank the National Natural Science Foundation of China (No.51541905) for its financial support of this work.

\section{REFERENCES}

[1] Marks P. "A sub takes to the skies-Imagine a craft that can soar like an eagle and swim like a stingray. Paul Marks meets the engineers with serious plans for an aircraft-submarine hybrid", New Scientist, Issue 2767, pp. 32-35, 2010. DOI:10.1016/S0262-4079(10)61629-6

[2] Edward D. "Flimmer: A Flying submarine", Spectra, Winter, pp. 6-9, 2014

[3] Chu X. S., Yan K., Wang Z., Zhang K., Feng G., Chen W.Q.: "Numerical simulation of water-exit of a cylinder with cavities", Journal of Hydrodynamics, Volume 5, Issue 22, pp.877-881, 2010. DOI:10.1016/S1001-6058(10)60045-5

[4] Cao W., You T.Q., Wei X.B., Wang C.,Zhao X.H., "Numerical Study on Air-involved Cavity during Water Exit of Underwater Vehicle", Procedia Earth and Planetary Science, Issue 5, pp. 203-208, 2012.

[5] Xu B.W., Feng J.F., Hu J.H., Liu A., Cheng X.D., "Dolphin-leap of the torpedo and the selection of the initial rotational angular velocity", Acta Physics. Sinica Volume 64, Issue 8, pp. (084501)1-11, 2015.

[6] Weiland C.J., Vlachos P.P., Yagla J.J., "Concept analysis and laboratory observations on a water piercing missile launcher”, Ocean Engineering, Issue 37, pp. 959, 2010. DOI:10.1016/j.oceaneng.2010.03.009

[7] Zink G., "Computational studies on the effect of water impact on an Unmanned Air Vehicle", MS Thesis, Iowa: Iowa State University, 2008.

[8] Goddard R., Eastgate J., "Submersible aircraft concept design study”, Final Report Arlington(VA): Naval Surface Warfare Center Carderock Division(US), Ship Systems Integration \& Design Department Report No.: NSWCCD-CISD-2010/011. OMB No.: 0704-0188, 2010 
[9] Kathryn W., "Submersible aircraft concept design study - amendment 1 (Additional assessment of design risks \& sensitivities within the original study, and an initial assessment of key control aspects)", Final Report Arlington(VA): Naval Surface Warfare Center Carderock Division(US), Ship Systems Integration \& Design Department Report No.: NSWCCD-CISD-2011/015. OMB No.: 0704-0188, 2011.

[10] Bašić J., Degiuli N., Werner A., "Simulation of Water Entry and Exit of a Circular Cylinder Using the ISPH Method", Transactions of FAMENA, Issue 38, pp. 45-62, 2014.

[11] Yan W.S., “Torpedo mechanics”, Xi'an China: Northwestern Polytechnical University Press, pp. 26-27, 2005.

[12] Fossen T., "Guidance and Control of Ocean Vehicles", 4th edn., West Sussex: John Wiley and Sons Ltd. p. 32-33, 1994.

[13] Логвиноовч Г В., “Гидродина Теченийсо Свободными Границами”, 1969. (translated by Shi H H) "Hydrodynamics of Free-Boundary Flows", Shanghai China: Shanghai Jiao Tong University Press p. 129-130, 2012.

[14] Li F.W., "Introduction to the air and gas dynamics" Xi'an China: Northwestern Polytechnical University Press, pp. 486-487, 2007.

[15] Chen B.L., "Theory and Algorithms of Optimization”, Beijing China: Tsinghua University Press, p256257, 2005.

Submitted: $\quad$ 08.5.2015

Accepted: $\quad$ 20.9.2016
Feng Jinfu

Li Yongli

$\mathrm{Xu}$ Baowei (corresponding author)

hkxubaowei@163.com

Liu An

Qi Duo

Engineering College of Aeronautics and Astronautics, Air Force Engineering University, Xi'an, 710038, China Flying instructor training base, Air Force Aviation University, Bengbu, 233000, China 